\title{
II. Nationalkomitee „Freies Deutschland“ und Entstehung ”wissenschaftlicher Forschungsinstitute“
}

Die Auflösung der Komintern machte den Weg frei zur Gründung des Nationalkomitees „Freies Deutschland“. Offizielle Begründung für die vollkommen überraschende Auflösung der Komintern war, daß der Zusammenschluß zur Internationale die Berücksichtigung der nationalen Besonderheiten des Kampfes gegen den Faschismus behindere. Nun sollte jede kommunistische Partei in eigener Verantwortung die jeweilige nationale Befreiungsbewegung in ihrem Heimatland unterstützen. In dieses Konzept paßte die Gewährung eines deutschen antifaschistischen Komitees auf sowjetischem Boden, das im Jahr zuvor noch abgelehnt worden war. Gleich nach Auflösung der Komintern wurde das NKFD ins Leben gerufen. Doch der Apparat der Komintern arbeitete unter der Tarnung „wissenschaftlicher Forschungsinstitute" ohne Unterbrechung weiter. Somit fiel die Komintern - wie sich die österreichische Kominternfunktionärin Ruth von Mayenburg erinnerte - nicht einfach auseinander: „Kriegsmäßig-konspirativ in einzelne ,Institute“ verpackt und mit Nummern versehen, wie ,Institut 205', ,Institut 100', ,Institut 99‘ und so weiter, entfaltete sie dann unter russischer Leitung eine mehr propagandistisch spezialisierte Tätigkeit."

\section{Institute 205 und 100}

Der Impuls zur Auflösung der Komintern kam am Abend des 8. Mai 1943. In einer Besprechung mit dem Volkskommissar für Äußere Angelegenheiten, Vjačeslav Molotov², kamen Dimitrov und Manuil'skij zu dem Schluß, „daß die Komintern als Führungszentrum für die kommunistischen Parteien unter den gegenwärtigen Bedingungen ein Hindernis für ihre selbständige Entwicklung und Erfüllung ihrer speziellen Aufgaben ist. Ein Schriftstück zur Auflösung dieses Zentrums wird erarbeitet. “3 Gemäß dieser Absprache wurde drei Tage später Stalin, Molotov und

1 Mayenburg, Blaues Blut und rote Fahnen, S. 298f. An anderer Stelle (Hotel Lux, S. $311 \mathrm{f}$.) spricht Mayenburg von der „Erbmasse [der Komintern] (...), aufgeteilt nach Aufgabenbereichen zwischen sogenannten "Instituten', von denen jedes eine Nummer erhielt." Leonhard (Revolution, S. 278) spricht von „einer Art Nachfolge-Organisation der Komintern“. Vgl. Nollau, Die Komintern, S. 64 ff.

2 Molotov war Volkskommissar für Auswärtige Angelegenheiten, vor allem aber war er enger Vertrauter Stalins, so daß er in vielerlei Hinsicht stellvertretend für Stalin agierte.

3 Dimitroff, Tagebücher, Eintrag vom 8. 5. 1943; vgl. Adibekov, Kominform, S. 6 ff.; Lebedeva/Narinskij, Rospusk Kominterna, S. $72 \mathrm{ff}$.; dies., Dissolution of the Comintern, S. $153 \mathrm{ff}$. Lebedeva und Narinskij folgen im wesentlichen Adibekovs Darstellung, übernehmen vor 
Georgij Malenkov eine Beschlußvorlage vorgelegt, in der die Begründung für die plötzliche Auflösung der Komintern insofern präzisiert wurde, als auf die sehr unterschiedlichen Bedingungen hingewiesen wurde, unter denen die jeweiligen kommunistischen Parteien den Widerstandskampf gegen den Faschismus in ihren Ländern zu führen hätten. Deswegen seien die organisatorischen Formen des Kampfes gegen den nach wie vor gemeinsamen Feind Hitler zu ändern und die Komintern aufzulösen ${ }^{4}$. Bot die von Dimitrov und Manuil'skij formulierte Vorlage noch keine konkreten Verfahrensvorschläge, so wurden diese auf der am 11. Mai stattfindenden Sitzung bei Stalin festgelegt. Mit dem vorbereiteten Beschlußentwurf war Stalin inhaltlich einverstanden, so daß gleich über das Procedere der Auflösung gesprochen wurde, nämlich - so die Eintragung Dimitrovs in sein Tagebuch -, "welche Funktionen [der Komintern] in welcher Form weitergeführt werden sollen" 5 . Die Auflösung der Komintern war bereits beschlossene Sache. Es ging nur noch um die organisatorische Umsetzung.

Das Exekutivkomitee der Komintern versammelte Dimitrov erst, nachdem die essentiellen Absprachen mit Stalin getroffen waren. Am 13. Mai 1943 wurde das EKKI mit der Auflösung der Komintern konfrontiert. Es nahm den vorgelegten Antrag jedoch einstimmig an. Immerhin wurde den EKKI-Mitgliedern bis zum 17. Mai Zeit gelassen, eventuelle Änderungsvorschläge zu machen. Noch kurz vor dieser Sitzung war Dimitrov von Stalin instruiert worden, das EKKI nicht mit der sowjetischen Entscheidung zu überrumpeln („Überstürzen Sie in dieser Angelegenheit nichts"). So erläuterte Dimitrov den Anwesenden, daß die Mitarbeiter des Kominternapparates weiterhin ihrer Arbeit in den Zeitungs-, Flugblatt- und Radioredaktionen nachgehen sollten, der Apparat der Komintern also mitnichten aufgelöst werde. Er erwähnte die Möglichkeit, daß „einzelne antifaschistische Komitees der Deutschen ins Leben gerufen werden [können]" 6 . Da diese Komitees aber noch gar nicht existierten, Dimitrov dazu offenbar auch keine näheren Erläuterungen gab, ging es vorerst darum, den Apparat trotz nomineller Auflösung der Komintern in seiner Funktionsfähigkeit zu erhalten. Darüber stimmte Dimitrov sich im weiteren mit der sowjetischen Führung ab. Zusammen mit einem Bericht über die Aufnahme des Auflösungsbeschlusses durch das EKKI übersandte er am nächsten Tag an Stalin und Molotov eine Bestandsaufnahme über die Einrichtungen der Komintern. Im Vordergrund standen technische Fragen wie die aktuelle perso-

allem die von ihm mehrfach zitierten Aussagen aus dem Dimitrov-Tagebuch, die Adibekov aber nicht belegt. Das vollständige Tagebuch ist bisher nur in bulgarischer Sprache veröffentlicht worden (Dimitroff, Dnewnik), aber für die Jahre 1933-43 liegt inzwischen eine deutsche Ausgabe vor, nach der hier zitiert wird.

4 Dimitrov und Manuil'skij an Stalin und Molotov am 11.5. 1943, RGASPI 495/73/174, Bl. 11.

5 Dimitroff, Tagebücher, Eintrag vom 11. 5. 1943; vgl. Posetiteli kremlevskogo kabineta, S. 67: anwesend waren Molotov, Dimitrov und Manuil'skij, Malenkov kam für die letzten zehn Minuten zu der Besprechung hinzu.

6 „Nicht den Eindruck vermitteln, daß wir die führenden ausländischen Genossen einfach davonjagen wollen. Sie werden für Zeitungen arbeiten. Es sollen vier Zeitungen gegründet werden (in deutscher, rumänischer, italienischer und ungarischer Sprache), ebenso können einzelne antifaschistische Komitees der Deutschen ins Leben gerufen werden usw.“, Dimitroff, Tagebücher, Eintrag vom 13.5. 1943. 
nelle Besetzung des Kominternapparates und die Zahl der Immobilien ${ }^{7}$. Am 17. Mai folgte auf einer zweiten EKKI-Sitzung die Endredaktion des Auflösungsbeschlus$\operatorname{ses}^{8}$, und auf einer dritten Sitzung am 19. Mai wurden die organisatorischen Fragen der Auflösung besprochen. Die Vorgabe war klar: „Alle diese Funktionen [des Kominternapparates] müssen in der ein oder anderen Form erhalten bleiben. Die neuen Bedingungen werden entsprechend der vom ZK der VKP (b) vorgegebenen Linie festgelegt." 9 Im Protokoll des EKKI hieß es daher, es seien Absprachen getroffen worden, „wie mit den wichtigsten Funktionen der Komintern verfahren werden muß, damit kein Schade für die Sache des Krieges gegen die räuberischen Hitleristen entstehe"10. Es wurde beschlossen, den Großteil aller Kominterneinrichtungen zu erhalten und in den ZK-Apparat der VKP (b) zu überführen. Am Abend des 19. Mai berichteten Dimitrov und Manuil'skij bei Stalin, der dabei mehrere Korrekturen an dem bereits vom EKKI redigierten Auflösungsbeschluß vornahm ${ }^{11}$.

Die Auflösung der Komintern wurde am 21. Mai abermals beschlossen, dieses Mal durch das Politbüro der VKP (b). Dem Politbüro gegenüber begründete Stalin den Schritt damit, daß es in Zeiten des Krieges nicht möglich sei, die internationale Arbeiterbewegung von einem Zentrum aus zu leiten. Jede kommunistische Partei fände in ihrem Land ganz unterschiedliche Bedingungen des antifaschistischen Kampfes vor. Während die deutschen und italienischen Kommunisten für den Sturz ihrer eigenen Regierungen kämpfen müßten, ginge es in anderen Ländern um die Befreiung von faschistischer Fremdherrschaft. Zudem wolle man mit der Auflösung der Komintern der Lüge entgegentreten, die kommunistischen Parteien seien Agenten Moskaus ${ }^{12}$. Die Pravda veröffentlichte den Auflösungsbeschluß am 22. Mai, und Stalin legte in einem exklusiven Interview mit der Nachrichtenagentur Reuter die Gründe für die Kominternauflösung nun öffentlich dar. Dabei betonte er besonders den außenpolitischen Kontext: „In jedem Land wird die kommunistische Partei eine Landespartei werden. In den alliierten Ländern wird diese nationale kommunistische Partei sich der Aufgabe widmen, die Kriegsanstrengungen nach Möglichkeit zu fördern. Die Initiative dürfte eine besonders gute Wirkung auf die Beziehung zwischen den Vereinigten Staaten und der Sowjetunion haben." 13

7 Dimitrov an Stalin und Molotov am 14. 5. 1943, RGASPI 495/73/174, Bl. 22; Dimitrov an Malenkov am 14. 5. 1943, ebenda B1. 27. In der Anlage befinden sich Berichte über die 1. Abteilung des EKKI (Presse und Propaganda), die Agentenausbildung der Komintern, das Informationsbulletin, die Immobilien sowie den Finanzhaushalt, ebenda Akte 182, Bl. 16-23 und 25-27. Am 17.5. 1943 reichte Dimitrov weitere Informationen an Malenkov nach, ebenda Akte 174, Bl. 47; vgl. Dimitroff, Tagebücher, Einträge vom 14. 5. und 17. 5. 1943.

${ }^{8}$ Dimitroff, Tagebücher, Eintrag vom 17. 5. 1943.

9 Ebenda, Eintrag vom 19. 5. 1943. Die angesprochenen Funktionen waren: „1) die nationalen Rundfunksendungen, 2) die Auslandsbüros der einzelnen Parteien, 3) die Aufrechterhaltung der Verbindung mit dem Ausland, 4) die Telegrafenagentur ,Surpress', 5) das Archiv, die Bibliothek, das Parteikomitee usw.".

10 Protokoll (B) Nr. 826 der geschlossenen Sitzung des EKKI-Präsidiums vom 19. 5. 1943, RGASPI 495/73/174, Bl. 51-55.

11 Dimitroff, Tagebücher, Eintrag vom 19. 5. 1943; vgl. Posetiteli kremlevskogo kabineta, S. 68.

12 Dimitroff, Tagebücher, Eintrag vom 21. 5. 1943.

13 Zitiert nach: Nollau, Die Internationale, S. 161 f.; vgl. „Antwort des Genossen Stalin auf die Frage des Hauptberichterstatters der englischen Presseagentur Reuter“, in: Stalin, Über den 
Stalins Hoffnung auf eine Verbesserung der alliierten Beziehungen hatte seine Bewandtnis darin, daß das ohnehin angespannte Verhältnis der Kriegsverbündeten im Frühsommer 1943 seinen Tiefpunkt erreicht hatte. Unmittelbarer Anlaß war die amerikanische und britische Entscheidung, den Termin zur Eröffnung einer zweiten Front in Westeuropa zu verschieben. Die Hauptlast des Krieges trugen nach zwei entbehrungsreichen Jahren immer noch allein die Rote Armee und die sowjetische Zivilbevölkerung. Die Beziehungen wurden zudem dadurch belastet, daß die Sowjetunion nicht auf Gebietsansprüche gegenüber Polen verzichtete. Bereits während der sowjetischen Besetzung Polens in den Jahren 1939-1941 war eine rigide Unterwerfungspolitik betrieben worden, die in den Massenerschießungen polnischer Offiziere durch das NKVD ihren Höhepunkt fand. Den Forderungen der polnischen Exilregierung in Großbritannien nach Aufklärung der in Katyn aufgedeckten Massengräber wollte die sowjetische Führung nicht nachkommen und brach am 25. April 1943 die diplomatischen Beziehungen zu Polen ab. Die sowjetischen Botschafter wurden Anfang Mai aus London und Washington abberufen. Und während es am 14. Mai auf dem Treffen Winston Churchills mit Theodore Roosevelt in Washington zu einer englisch-amerikanischen Annäherung kam, berichtete die sowjetische Presse über das Ende der Komintern und den Beginn des nationalen Befreiungskampfes der vom Faschismus unterdrückten Völker. Bereits am 8. Mai war die Existenz eines „Bundes Polnischer Patrioten in der UdSSR“ sowie die zukünftige Aufstellung einer polnischen Befreiungsarmee in der Sowjetunion bekanntgegeben worden ${ }^{14}$. Selbst wenn in der historischen Rückschau die Gründe für die Auflösung der Komintern als „durchsichtig“ und „Täuschung“ tituliert werden ${ }^{15}$, der Zeitpunkt der Auflösung war genau auf die Bedürfnisse der sowjetischen Außenpolitik abgestimmt.

Die weiteren Schritte zur Überführung des Kominternapparates in neue Organisationsformen wurden unmittelbar nach dem Politbürobeschluß der VKP (b) unternommen. Noch am Abend des 21. Mai rief Dimitrov die Abteilungsleiter des Kominternapparates zu sich und stimmte sie auf das Kommende ein ${ }^{16}$. Am 24. Mai unterbreitete er den Spitzen der deutschen, ungarischen, rumänischen und italienischen Exil-KP, daß nun anstelle der Kommunistischen Internationale nationale antifaschistische Komitees den Kampf gegen den Faschismus führen würden. Diese sollten aus „Persönlichkeiten des öffentlichen Lebens und bekannten kriegsgefangenen Antifaschisten“ gebildet werden. Jede Partei wurde aufgefordert, entsprechende Vorschläge auszuarbeiten ${ }^{17}$. In den folgenden zwei Wochen traf Dimitrov wiederholt Absprachen mit Manuil'skij, wie die Schaffung antifaschistischer Komi-

Großen Vaterländischen Krieg, S. $85 \mathrm{ff}$; siehe auch Dimitroff, Tagebücher, Einträge vom 22. 5. und 29. 5. 1943.

14 Lebedeva/Narinskij, Dissolution of the Comintern, S. 159f.; vgl. Bonwetsch, Der „Große Vaterländische Krieg“, S. 981 ff.; Gosztony, Stalins fremde Heere, S. $78 \mathrm{ff}$.

15 Ulam, Expansion and Coexistence, S. 346: „Der wahre Grund für die Auflösung der Komintern war ein wenig anders als der angegebene: Die Hörigkeit gegenüber Moskau und die Maschinerie diese zu erzwingen wurde jetzt in jede größere kommunistische Partei eingepflanzt, und es gab keinen Bedarf mehr an äußerlichen Formen der Einheit. (...) In der Rückschau waren die Gründe für die Auflösung der Komintern durchsichtig und können unter dem Oberbegriff ,Täuschung' gefaßt werden."

16 Dimitroff, Tagebücher, Einträge vom 21. 5. und 22. 5. 1943.

17 Ebenda, Eintrag vom 24. 5. 1943. 
tees auf den Weg zu bringen sei ${ }^{18}$. Zu der in organisatorischer Hinsicht entscheidenden Sitzung kam es am 12. Juni bei Stalin: Die geplanten nationalen antifaschistischen Komitees sollten von einer „Abteilung Internationale Information“ (otdel meždunarodnoj informacii - OMI) angeleitet werden, die im Apparat des ZK der VKP (b) neu geschaffen werden sollte, um die Funktionen der aufgelösten Komintern zu übernehmen. Auf eben dieser Sitzung wurde auch die Gründung des Nationalkomitees „Freies Deutschland" beschlossen ${ }^{19}$.

Der Beschluß über eine eigenständige ZK-Abteilung zur Übernahme des alten Kominternapparates wurde zwei Tage später wieder verworfen. Statt der ursprünglich vorgesehenen ZK-Abteilung unter Leitung von Ščerbakov wurde lediglich innerhalb der Geschäftsabteilung des ZK der VKP (b) ein "Sonderapparat" zur Sicherstellung der technischen und materiellen Voraussetzungen der Kominterngeschäfte geschaffen. An Stelle der früheren Sektionen der Komintern wurden sogenannte Auslandsbüros der kommunistischen Parteien eingesetzt, die den Kontakt zu ihren im Heimatland (im Untergrund) operierenden Parteien über geheime Funkverbindungen halten und ihre Anweisungen über von Moskau aus ausgestrahlte Radiosendungen geben sollten. Dazu entschied eine Kommission zur Liquidierung der Komintern ${ }^{20}$, „das Radiozentrum mit seinen Objekten [Funkstationen], das Studio für den nationalen Rundfunk und die Bibliothek zur Nutzung durch die Auslandsbüros der kommunistischen Parteien und zur Aufrechterhaltung der Funkverbindung mit dem Ausland zu erhalten"21. Alle Immobilien des EKKI wurden der Geschäftsabteilung des ZK der VKP (b) überstellt. Das Archiv sowie die Personalunterlagen der Kominternfunktionäre übernahm die Kaderabteilung des ZK der VKP (b). Innerhalb der Geschäftsabteilung des ZK entstand der vorgeschlagene "Sonderapparat", der in einem „besonderen Stellenplan“ die Mitglieder der Auslandsbüros als Mitarbeiter führte. Lediglich die Kaderschulung der Komintern, zuletzt in einem „Objekt“ in Kušnarenkovo durchgeführt, wurde vollständig eingestellt ${ }^{22}$.

Im Juni 1943 entschied sich die sowjetische Führung für eine dezentrale Fortführung der Kominterngeschäfte, was zu der „kriegsmäßig-konspirativen Verpackung

18 Ebenda, Einträge vom 29. 5. und 4. 6. 1943.

19 Ebenda, Eintrag vom 12. 6. 1943; vgl. Posetiteli kremlevskogo kabineta, S. 71, anwesend waren: Stalin, Molotov, Berija, Malenkov, Vorošilov, Ščerbakov, Dimitrov und Mikojan.

20 Der Kommission gehörten an: Dimitrov (Vorsitzender), Manuil'skij, Pieck, Togliatti und Sucharev (Chef der EKKI-Wirtschaftsabteilung), Protokoll (B) Nr. 828 der geschlossenen Sitzung des Präsidiums des EKKI vom 8. 6. 1943, RGASPI 495/73/174, Bl. 67-68; Dimitroff, Tagebücher, Eintrag vom 8. 6. 1943; vgl. Nollau, Die Internationale, S. 164.

21 Undatierter Beschluß der Kommission zur Liquidierung der Kommunistischen Internationale, RGASPI 495/73/174, Bl. 78.

22 „Auskunft über die Anzahl der Mitarbeiter in dem geplanten Stellenplan“ vom 19. 7. 1943 und „Stellenplan für den Sonderapparat innerhalb der Geschäftsabteilung des ZK der VKP (b)“ vom 21. 7. 1943, RGASPI 495/73/208, Bl. 110-120. Leider können hier nur die Entwürfe zitiert werden, da andere Archivdokumente für die Benutzung gesperrt sind. Über das tatsächliche Zustandekommen der Planungen besteht jedoch kein Zweifel. Inhaltlich stimmen die hier dargelegten Beschlüsse mit den zusammen mit Malenkov am 31.5. 1943 getroffenen Vorabsprachen Dimitrovs überein. Vermutlich wurde der oben zitierte Beschluß der Liquidierungskommission am 12. Juni gefaßt, am Abend desselben Tages von Stalin korrigiert und anschließend am 14. Juni endgültig verabschiedet, vgl. Dimitroff, Tagebücher, Einträge vom 31.5. und 12. 6. 1943; Adibekov, Komintern, S. 10. 
in Institute " führte ${ }^{23}$. An Stelle einer eigenständigen ZK-Abteilung wurde das nach außen hin selbständig agierende „Institut 205“ und das „Institut 100“ mit jeweils eigenem Stellenplan gegründet. Die ursprünglich als weitere Aufgabe des „Sonderapparates" vorgesehene Anleitung der neu zu schaffenden antifaschistischen Komitees - die spätere Funktion des „Instituts 99 “ - wurde vorerst fallengelassen, weil diese Komitees noch nicht existierten ${ }^{24}$. Das Fehlen einer übergeordneten Instanz, wie sie mit der „Abteilung Internationale Information“ geplant gewesen war, hatte auf die institutionelle Einbindung der „Institute“ keinerlei Auswirkungen. Durch die bestehenden personellen Verflechtungen blieben die Kontroll- und Anleitungsmechanismen bestehen, vor allem durch das „Büro Dimitrov“, das seine Arbeit bis zur Schaffung des OMI im Juli 1944 fortsetzte ${ }^{25}$. Das scheinbar eigenständige Agieren sowie die Tarnbezeichnung als „wissenschaftliche Forschungsinstitute “ förderte den Eindruck einer vollständig vollzogenen Auflösung der Komintern.

Herzstück der aufgelösten Komintern waren die Redaktionen und Sender der ehemaligen Presse- und Propagandaabteilung des EKKI. Seit dem 1. September 1943 arbeiteten sie, ohne je ihre Tätigkeit unterbrochen zu haben, unter der Bezeichnung "Institut 205“ weiter, die bereits während der Evakuierung des Kominternapparates nach Ufa benutzt worden sein soll26. Ansonsten läßt sich die Numerierung der Institute weder durch konkrete Zusammenhänge noch durch eine Zahlensymbolik motivieren. Leiter des Instituts 205 war der tschechische Kommunist Friedrich Geminder, der schon die Presse- und Propagandaabteilung geleitet hatte. Die bisherigen Aufgaben blieben bestehen: Aufrechterhaltung des Sendebetriebs und politische Kontrolle der Redaktionen, Herausgabe eines wöchentlichen Informationsbulletins, Pressearchiv und Radioabhördienst ${ }^{27}$. Im Institut 205 gab es eine österreichische, ungarische, bulgarische, rumänische, finnische, jugoslawische, spanische, französische, polnische, tschechische, slowakische, sudetendeutsche, italienische, norwegische und vier deutsche Redaktionen ${ }^{28}$. Während des Krieges blieb die Zahl der Mitarbeiter trotz einiger personeller Wechsel konstant ${ }^{29}$. Nach dem Kriegsende wurden die Radiosender des Instituts 205 eingestellt. Nur die Presseauswertung und der Radioabhördienst liefen weiter, standen aber mehr und mehr in Konkurrenz zum sowjetischen Nachrichtenbüro TASS (Telegrafnoe agenstvo So-

23 Mayenburg, Blaues Blut und rote Fahnen, S. $298 \mathrm{f}$.

24 "Vorschlag in der Frage der Liquidierung der Komintern" vom 17.6. 1943, RGASPI 495/73/174, Bl. 69-73.

25 Foitzik, Zur Anleitung der kommunistischen Parteien, S. 509 ff.; vgl. den Bestand „Büro Dimitrov" im RGASPI, Kratkij putevoditel', S. 82.

26 Erinnerungen Richard Gyptners, SAPMO-BArch SgY 30/0331, Bl. 80; vgl. Nollau, Die Komintern, S. 65.

27 Tätigkeitsbericht des Instituts 205 für die Zeit vom 1.9. 1943 bis 1.2. 1944, RGASPI 495/10a/433e, Bl. 49-50.

28 Geschäftsverteilungsplan für das Institut 205 (undatierter Entwurf), RGASPI 495/73/208, Bl. 150-154; siehe auch Gyptner, Über die antifaschistischen Sender, S. 881 ff.; Kraushaar, Zur Tätigkeit und Wirkung des „Deutschen Volkssenders“, S. 116 ff.; vgl. Mayenburg, Hotel Lux, S. $283 \mathrm{ff}$.

29 Zum 1. September 1943 waren es 209 und zum 1. Februar 1944, inklusive einiger personeller Wechsel, 210 Personen; Geschäftsverteilungsplan des Instituts 205 vom Sommer 1943 und Tätigkeitsbericht vom Februar 1944, RGASPI 495/73/208, Bl. 150-154 und ebenda 495/10a/433e, Bl. 49-50; zur Arbeit im Institut 205 siehe den Bericht der ehemaligen Institutsmitarbeiterin (ab Herbst 1944) Genia Quittner, Weiter Weg nach Krasnogorsk, S. $265 \mathrm{f}$. 
vetskogo Sojuza), das schließlich gegen Ende 1946 diese Dienste übernahm. War bereits durch das Kriegsende ein maßgeblicher Teil der Aufgaben - die antifaschistischen Sender - verloren gegangen, so verlor das Institut 205 durch die Rückkehr der Emigranten in ihre Heimatländer nach und nach auch seine Mitarbeiter. Um die Jahreswende 1946/47 wurde der Stellenplan des Instituts, der zwischenzeitlich 326 Mitarbeiter umfaßt hatte, um beinahe die Hälfte auf 177 Mitarbeiter zusammengestrichen. Im Februar 1948 schließlich wurde das Institut 205 aufgelöst. Die Reste seines ausländischen Mitarbeiterstabes wurden dem Institut 100 zugeschlagen ${ }^{30}$.

Das Institut 100 hatte bei der Auflösung der Komintern ohne wesentliche Änderungen die 1. Abteilung des EKKI übernommen. Diese war der sogenannte geheime operative Dienst zur Ausbildung von Kominternagenten, die zur Aufrechterhaltung des Kontaktes der Exil-Parteileitungen zu ihren im Heimatland im Untergrund agierenden Mitgliedern in die jeweiligen Länder eingeschleust wurden ${ }^{31}$. Die Komintern versuchte damit, ein weltweites Netz von über Funk angeleiteten Agenten aufzubauen. Innerhalb Europas war dieses Netz aber offenbar sehr löchrig. So hatte die Funkstation in England - vermutlich aus Rücksicht auf den Kriegsverbündeten - den Betrieb eingestellt, und die Funkstationen innerhalb des Großdeutschen Reiches galten als „im Aufbau befindlich“. In Ungarn und Bulgarien gab es gar keine Stationen ${ }^{32}$. Im Sommer 1943 wurde entschieden, die technischen Einrichtungen unter der Tarnbezeichnung „Institut 100 “ beizubehalten „zum Zwecke der Aufrechterhaltung der Funkverbindung der in Moskau befindlichen Auslandsbüros der kommunistischen Parteien mit ihren Heimatländern, sowie der Funkverbindung mit den speziellen Funkstationen im Ausland“33. Leiter des Instituts 100 wurde Ivan Andreevič Morozov, ein Abteilungsleiter aus der NKVD-Verwaltung für Aufklärung ${ }^{34}$.

Das Institut 100 übernahm eine Schule, in der die Agenten neben der obligatorischen politischen Schulung eine Ausbildung zum Funker und eine technische Einweisung in den Bau von Behelfsfunkgeräten erhielten. Diese sogenannte „Techschule" (technische Schule) befand sich in Puškino, 30 Kilometer nordöstlich von Moskau. Zum Zeitpunkt der Auflösung der Komintern hielten sich dort 50 Schüler auf, von denen ein Teil noch im Mai/Juni 1943 in Ungarn, der Slowakai, Polen, Finnland, Österreich und Rumänien eingesetzt wurde ${ }^{35}$. Außer der technischen

30 Adibekov, Kominform, S. 12 ff. Das Institut 205 stand seit Juli 1946 unter der Leitung des sowjetischen ZK-Funktionärs N.N. Puchlov.

31 Analog zu diesen Aufgaben gab es sechs sogenannte Sektoren: (1) Anleitung der Auslandspunkte, (2) Fälscherwerkstätten, (3) Vorbereitung der Agenten, (4) Chiffrierabteilung, (5) Wirtschaftsabteilung und (6) Funkstation. Die Funkstation war noch einmal untergliedert in fünf sogenannte Objekte: (1) Sendestation, (2) Empfangsstation, (3) Werkstatt zum Bau von Funkgeräten, (4) technische Abteilung zur Wartung der Anlagen, (5) ReserveRadiostation in Ufa und für die Verbindung mit dem Fernen Osten eine Relaisstation in Alma-Ata, Tätigkeitsbericht der 1. Abteilung des EKKI vom 14.5.1943, RGASPI 495/73/182, Bl. 16-23.

32 Tätigkeitsbericht der 1. Abteilung des EKKI vom 14. 5. 1943, RGASPI 495/73/182, Bl. 21-22.

33 Planungspapier über die Einrichtung des Instituts 100, RGASPI 495/73/174, Bl. 81; vermutlich als Anlage zu dem Schreiben Dimitrovs an Ščerbakov vom 14. 7. 1943, ebenda Akte 182, Bl. 28.

34 Dimitrov an Malenkov am 22. 5. 1943, RGASPI 495/73/182, Bl. 1; vgl. Lubjanka, S. 272.

35 Lebedava/Narinskij, Dissolution of the Comintern, S. 157. 
Schule gab es die "Guljaev-Gruppe“ in dem ebenfalls im Moskauer Umland gelegenen Nagornoe. Dort folgten nach Abschluß der technischen Ausbildung Unterweisungen in Theorie und Praxis der Diversion, Kurse über die Taktik der sowjetischen Partisaneneinheiten, Anleitung zum Häuserkampf, Seminare zum Aufbau eines illegalen Parteiapparates und Vorträge über die „aktuelle Politik und die internationale Lage“. Für die Lehrveranstaltungen wurden Militärspezialisten bzw. Kominternfunktionäre verpflichtet ${ }^{36}$. Das Schulungslager stand unter der Leitung des sowjetischen Funktionärs Pantalejmon Guljaev und wurde, da sich die ausgebildeten Agenten bis zu ihrem Einsatz mitunter länger in Nagornoe aufhielten, auch „Reservegruppe der Bruderparteien“ genannt. Das Camp diente sowohl als Vorbereitungslager als auch als Wartestation. Die Einsätze selbst kamen Partisanenaktionen gleich und erfolgten in Absprache mit der militärischen Aufklärung (GRU) bzw. dem militärischen Geheimdienst (SMERŠ) ${ }^{37}$.

Mittels der Institute 205 und 100 konnten die Geschäfte der aufgelösten Komintern ohne Unterbrechung fortgeführt werden. Ein drittes Institut wäre nicht notwendig gewesen. Aber mit der Gründung des Nationalkomitees „Freies Deutschland" entstand eine neue Aufgabe, die über die bisherige Tätigkeit der Komintern hinausging.

\section{Nationalkomitee „Freies Deutschland“}

Die Gründung des Nationalkomitees „Freies Deutschland“ stand in unmittelbarem Zusammenhang mit der Auflösung der Komintern, wurde aber erst in die Wege geleitet, als der Erhalt der Funktionsfähigkeit des Kominternapparates gesichert war. Um die „Frage des Komitees", so notierte sich Wilhelm Pieck in der Besprechung Dimitrovs mit den führenden EKKI-Funktionären, ging es erst am 24. Mai 1943. Nach dem bereits in der Antifa eingeübten Muster sollte es auf einer Konferenz von Kriegsgefangenen aus der Taufe gehoben und ,aus kriegsgefangenen deutschen Offizieren und Soldaten und in der Sowjetunion lebenden deutschen Hitlergegnern Schriftsteller, Gelehrte, Künstler, Politiker" zusammengesetzt werden. Als Aufgabe wurde der „Zusammenschluß aller Hitlergegner zur kämpfenden Einheit für die Rettung des deutschen Volkes aus der Katastrophe" genannt ${ }^{38}$. Auf der Grundlage dieser Absprache formulierte die KPD den „Vorschlag zur Bildung des deutschen Komitees zum Kampf gegen Hitlerkrieg und Nazityrannei“, den Dimitrov am 27. Mai 1943 erhielt ${ }^{39}$. Nachdem Dimitrov am 1. Juni mit den deutschen Kommuni-

36 Pliševskij an Dimitrov am 2. 8. 1943 über die Ausbildung einer Gruppe von 35 Absolventen der Kominternschule in Kušnarenkovo, RGASPI 495/73/176, B1. 58.

$37 \mathrm{Vgl}$. Untersuchungsbericht von Manuil’skij an Ščerbakov vom 10. 11. 1943 über den gescheiterten Einsatz einer rumänischen Kominterngruppe, RGASPI 17/125/183, Bl. 189-192. Die alleinige Zuständigkeit für den Einsatz von Kominternagenten hatte Morozov gegenüber Guljaev durchgesetzt, um parallele Strukturen zu vermeiden; Morozov an Dimitrov am 15. 3. 1943, RGASPI 495/73/176, Bl. 21; zur Arbeitsweise der militärischen Geheimdienste siehe Glantz, Soviet Military Intelligence, S. $202 \mathrm{ff}$.

38 Notizen Piecks über die Besprechung bei Dimitrov am 24. 5. 1943 über die „Frage des Komitees“, SAPMO-BArch NY 4036/575, Bl. 7-8; vgl. Dimitroff, Tagebücher, Eintrag vom 24. 5.1943.

39 SAPMO-BArch NY 4036/575, Bl. 3-5; vgl. die Notizen Piecks zur „Frage des Komitees“, 
sten Rücksprache gehalten hatte, arbeitete er den KPD-Entwurf um zu einer Beschlußvorlage für das ZK der VKP (b) und schickte sie am 11. Juni 1943 an Stalin ${ }^{40}$. Dabei machte er deutlich, daß das deutsche Komitee Modellcharakter für weitere Komitees Kriegsgefangener anderer Nationalitäten haben könnte: „Ich schicke Ihnen beiliegenden Entwurf für einen Beschluß des ZK der VKP (b) über die Gründung des antifaschistischen Komitees ,Freies Deutschland', den wir unter Berücksichtigung der Vorschläge des Genossen Pieck und anderer deutscher Genossen ausgearbeitet haben. Ich denke, daß im folgenden auf ähnlicher Grundlage auch ein ungarisches, italienisches und rumänisches antifaschistisches Komitee gegründet werden kann." 41

Am 12. Juni 1943 wurde vom Politbüro der VKP (b) die Gründung eines deutschen antifaschistischen Komitees beschlossen. Dabei wurde auch der Name Nationalkomitee „Freies Deutschland“ festgesetz ${ }^{42}$. In den Vorschlägen der KPD waren noch die Bezeichnungen „Deutsches Komitee zum Kampf gegen Hitlerkrieg und Nazityrannei“ oder "Deutsches Antihitler-Komitee" verwendet worden ${ }^{43}$. Auch wenn sich in den Beschlüssen bzw. in den Notizen Piecks keinerlei Hinweise darauf finden lassen, wie die Wahl des Namens motiviert war, so liegt der Bezug zu der im Januar 1942 von deutschen Kommunisten im mexikanischen Exil ins Leben gerufenen „Bewegung Freies Deutschland“ auf der Hand. Durch die Wahl des gemeinsamen Namens wurde zumindest erreicht, daß westliche Beobachter an innere $\mathrm{Zu}$ sammenhänge glaubten und somit das propagandistische Auftreten der deutschen Kriegsgefangenen in der Sowjetunion unter dem Namen Nationalkomitee „Freies Deutschland" größeres Gewicht bekam ${ }^{44}$. Ein tatsächlicher Zusammenhang mit der weltweiten Bewegung „Freies Deutschland“ ist in den - verfügbaren - sowjetischen Quellen jedoch nicht zu erkennen. Auch spielte die internationale Bewegung bei den innersowjetischen Diskussionen keine Rolle. Die Entscheidung zur Gründung des "Anti-Hitler-Komitees“, obgleich unter der Bezeichnung Nationalkomitee „Freies Deutschland“, rekurrierte ausschließlich auf den vom Büro für militärpolitische Propaganda im April 1942 vorgelegten Plan, der an das sowjetische Konzept antifaschistischer Komitees unter Regie des ZK der VKP (b) anknüpfte. Indiz für eine derartige Interpretation ist die Bemerkung Piecks, die dieser bei der Ablage der von der KPD ausformulierten Beschlußvorlage zur Komiteegründung machte: „Plan im Frühjahr '42 nach Rückkehr aus Ufa, 27. 5. 43 an Dimitroff." 45

ebenda, Bl. 7-8. Piecks Notizen tragen das Datum 27. 5. 1943, das sich aber auf den von der KPD ausgearbeiteten Vorschlag und nicht auf den Zeitpunkt der Niederschrift beziehen dürfte. Wahrscheinlich machte sich Pieck die Notizen während oder nach der Besprechung mit Dimitrov. Anhand dieser Stichpunkte wurde dann das KPD-Papier geschrieben.

40 Dimitroff, Tagebücher, Einträge vom 1. 6. und 11.6.1943.

41 Dimitrov an Stalin am 11.6. 1943, RGASPI 495/77/155, Bl. 107. Eine Kopie des Briefes schickte Dimitrov am 11. 6. 1943 an Malenkov, ebenda, Bl. 108. Die Anlagen zu beiden Briefen befinden sich nicht in der Akte.

42 Dimitroff, Tagebücher, Eintrag vom 12. 6. 1943.

43 Notizen Piecks von der Besprechung bei Dimitrov am 24. 5. 1943, SAPMO-BArch NY 4036/575, Bl. 7.

44 Bungert, Das Nationalkomitee und der Westen, S. $68 \mathrm{ff}$.; vgl. Mühlen, Fluchtziel Lateinamerika, S. $124 \mathrm{ff}$.

45 „Vorschlag zur Bildung des deutschen Komitees zum Kampf gegen Hitlerkrieg und Nazityrannei“, SAPMO-BArch NY 4036/575, Bl. 3. 
Der Inhalt des Politbürobeschlusses zur Gründung des Nationalkomitees „Freies Deutschland" läßt sich lediglich über die Notizen Piecks vom 16. Juni rekonstruieren, als er von Dimitrov über die Entscheidung informiert wurde: „Aufgabe des Komitees [ist die] allseitige Unterstützung des Kampfes des deutschen Volkes gegen Hitler durch massenhaft schriftl[iche] und Radiopropaganda für [die] unverzügliche Beendigung des Hitlerkrieges mittels Sturz der Hitlerclique und Schaffung eines freien Deutschlands (...) Zahl der Mitglieder des Komitees - 33, davon 22 von Kriegsgefangenen und 11 von antifasch[istischen] Kämpfern; als Präsident des Komitees - Erich Weinert, als Ehrenpräsident - Heinrich Mann, wenn dieser einverstanden." 46 Noch am 16. Juni 1943 wurde ein vorbereitender Ausschuß nominiert ${ }^{47}$. Je zwei Instrukteure - ein KPD-Funktionär und ein kriegsgefangener Absolvent der Antifa-Schule - fuhren im Juni in die (Mannschafts-)Kriegsgefangenenlager, um dort Delegierte zur Gründung des NKFD auszuwählen ${ }^{48}$. Gesondert davon war bereits seit dem 20. Mai eine Gruppe Politoffiziere der Politischen Hauptverwaltung mit der Werbung der Offiziere für das Nationalkomitee beschäftigt, was darauf hindeutet, daß es noch vor dem hier dargestellten Prozeß der Beschlußfassung unter Einbindung der KPD eine Abstimmung der sowjetischen Entscheidungsträger gegeben haben muß. Die KPD wurde erst am 24. Mai instruiert. So bezeichnete Pieck in der Rückschau die Gründung des NKFD als „relativ schnelle Entwicklung“, die er in einem Vortrag vor Mitgliedern der Exil-KPD in Moskau kritisch bewertete: „Vorarbeit in den Lagern und Schulen, aber etwas eng. Antifa mehr für Soldatenmassen, nicht geeignet [für] Offiziere - besonders höhere Offiziere. Aber Notwendigkeit, höhere Offiziere zu gewinnen; bisher nur wenige (Hadermann, Reyher, Charisius, Augustin, Kügelgen). Besonders seit Stalingrad 22 Generäle, 170 Offiziere im Offizierslager, in anderen Lagern. Ende Mai 1. Brigade im Offizierslager - Schwierigkeiten, ich [am] 18. 6. dorthin."49

Das Problem bei der Vorbereitung des Nationalkomitees war, höhere Offiziere und Generäle zur Mitarbeit zu gewinnen, ohne deren Beteiligung Dimitrov das Komitee nicht gründen wollte ${ }^{50}$. Allerdings blieb die in das Offizierslager Suzdal' (Lager Nr. 160) entsandte Delegation der 7. Verwaltung des GlavPURKKA ohne Erfolg51. Auch die Bemühungen des UPVI-Chefs Petrov und des Chefs der 7. Ver-

46 „Entwurf zum Beschluß über [die] Schaffung eines Nationalen Komitees Freies Deutschland“, Notizen Piecks vom 16.6. 1943, SAPMO-BArch NY 4036/575, Bl. 10-12. Nach Gründung des NKFD konzentrierten sich die sowjetischen Werbungsversuche mehr auf Thomas Mann, siehe S. 169, Anm. 158.

47 Von kommunistischer Seite gehörten ihm an: Johannes R. Becher, Hans Mahle, Wilhelm Pieck, Walter Ulbricht und Erich Weinert; von den Kriegsgefangenen: Gefreiter Jakob Eschborn, Hauptmann Ernst Hadermann, Leutnant Bernt v. Kügelgen und Feldwebel Herbert Stresow; Weinert, Nationalkomitee, S. 17. Anstelle der Kriegsgefangenen waren ursprünglich vorgesehen: Willi Bredel, Fritz Erpenbeck, Otto Winzer und Friedrich Wolf; vgl. die Notizen Piecks vom 16.6. 1943: „später geändert, daß auch Kriegsgefangene in Initiativgruppe“, SAPMO-BArch NY 4036/575, Bl. 11.

48 Liste der Instrukteure, SAPMO-BArch NY 4036/571, Bl. 75.

49 Notizen Piecks für einen am 16. 7. 1943, nach der Gründung des NKFD gehaltenen Vortrag vor den in Moskau lebenden KPD-Mitgliedern, SAPMO-BArch NY 4036/575, Bl. 54.

50 Notizen Piecks über die Besprechung bei Dimitrov am 24. 5. 1943 zur „Frage des Komitees", SAPMO-BArch NY 4036/575, Bl. 8.

51 Vgl. Ulbricht an Dimitrov am 20.5. 1943 über die Abfahrt der Delegation, RGASPI 
waltung Burcev, die zusammen mit Pieck am 18. Juni ebenfalls nach Suzdal' fuhren, blieben ergebnislos. $\mathrm{Da}$ sich die Generäle nicht gesprächsbereit zeigten, widmete sich die Kommission der „Obersten- und Major-Abteilung“, die „bisher von der Arbeit nicht erfaßt" worden war. Aber selbst diese Bemühungen scheiterten. Pieck kam zu dem Schluß: „Offiziere sehen es als taktlos an, sie so schnell zur Entscheidung zu drängen. “52 An Manuil'skij berichtete er: „Natürlich war sowohl bei den Gesprächen wie bei der Versammlung immer die zur Entscheidung stehende Frage der Gewinnung der Leute für die Bildung eines Nationalkomitees die Hauptsache und der Nachweis, daß dieses Komitee dem deutschen Volk helfen soll, dem Krieg ein Ende zu machen und die Folgen der weiteren Fortsetzung des Krieges zu verhindern. Aber dieser Gedanke ist bisher noch wenig in ihre Köpfe eingegangen. Die Frist, das den Leuten auseinanderzusetzen und eine wirkliche innere Umstellung bei ihnen hervorzurufen, war zu kurz. " 53 Die in Einsiedels Erinnerungen erwähnten „endlosen Diskussionen“ zwischen den Offizieren und den deutschen Kommunisten bestätigen den in Suzdal' von Pieck und Arnold formulierten Eindruck, daß für eine gemeinsame Gründung des Nationalkomitees die Voraussetzungen fehlten $^{54}$.

Obwohl die eigentliche Zielgruppe, die höheren, zumeist in Stalingrad in Gefangenschaft geratenen Offiziere, nicht erreicht werden konnte, wurde am Zeitplan, den die Vorlage zur Komiteegründung mit „spätestens in eineinhalb bis zwei Monaten" gesteckt hatte ${ }^{55}$, unbeirrt festgehalten. Das Gründungsmanifest des NKFD wurde „pünktlich“ am 12. Juli in Krasnogorsk von 12 Kommunisten und 21 Kriegsgefangenen unterzeichnet ${ }^{56}$. Betrachtet man die Zusammensetzung des Nationalkomitees, so waren die sowjetischen Politoffiziere und die deutschen Kommunisten mit diesem Ergebnis weit hinter den selbst gesteckten Zielen zurückgeblieben. Die Generäle, insbesondere der heftig umworbene Generalfeldmarschall Paulus, waren dem NKFD gänzlich ferngeblieben. Immerhin hatte man sechs Stalingrad-Offiziere gewinnen können. Aber die ranghöchsten Komiteemitglieder waren lediglich die drei Majore Karl Hetz, Heinrich Homann und Herbert Stößlein. Ein propagandistischer Glücksfall war der über Stalingrad abgeschossene Fliegerleutnant Heinrich Graf von Einsiedel, der ein Urenkel des Reichskanzlers Otto von Bismarck war, und sich sehr schnell dem NKFD angeschlossen hatte. Im Kern aber stützte sich der Offiziersflügel des NKFD auf Haupmann Hadermann, die Oberleutnante Charisius, Reyher und Rücker sowie Leutnant Ernst Kehler, die seit Mai 1942 der soge-

495/77/27, Bl. 93; Protokolle über die Befragungen im Lager Nr. 160 in der Zeit vom 1. bis 25. 6. 1943, SAPMO-BArch NY 4036/571, B1. 81-109.

52 Piecks „Notizen über den Besuch des Offiziers-Kriegsgefangenenlagers in Susdal vom 18.-28. 6. 1943“, SAPMO-BArch NY 4036/498, Bl. 72-82.

53 Pieck an Manuil'skij am 21. 6. 1943, SAPMO-BArch NY 4036/571, Bl. 110+RS.

54 Einsiedel, Tagebuch der Versuchung, S. 61; vgl. die demgegenüber beschönigende Schilderung bei Blank, Die Zusammenkunft Wilhelm Pieck mit kriegsgefangenen Generalen und Offizieren, S. $675 \mathrm{ff}$.

55 „Vorschlag zur Bildung des deutschen Komitees zum Kampf gegen Hitlerkrieg und Nazityrannei“", SAPMO-BArch NY 4036/575, Bl. 4.

56 In dem KPD-Vorschlag vom 27. 5. 1943 gab es noch zwei Varianten: 33 Mitglieder (11 Zivilisten und 22 Kriegsgefangene) oder 22 Mitglieder in paritätischer Besetzung, SAPMOBArch NY 4036/575, Bl. 4. 
nannten antifaschistischen Offiziersgruppe angehörten ${ }^{57}$. Auch bei den Mannschaftsdienstgraden stützte man sich auf die Aktivisten, die seit den Anfängen der Antifa dabei waren. Fast alle waren seit mindestens einem Jahr in sowjetischer Gefangenschaft. Sieben von ihnen hatten unterdessen eine Antifa-Schule besucht. Fünf von den zehn unterzeichnenden Soldaten waren zudem Kommunisten, die gezielt zur Roten Armee übergelaufen waren.

Kriegsgefangene Mitglieder des NKFD 58

\begin{tabular}{|c|c|c|c|}
\hline Dienstgrad & Name & Gefangenschaft & Bemerkungen \\
\hline \multirow[t]{4}{*}{ Major } & Hetz & Jan. 43 & Stalingrad \\
\hline & Homann & Jan. 43 & Stalingrad \\
\hline & Krausnick & 5.12 .42 & \\
\hline & Stößlein & Jan. 43 & Stalingrad \\
\hline \multirow[t]{2}{*}{ Hauptm. } & Fleischer & Jan. 43 & SPD, Stalingrad \\
\hline & Hadermann & 18. 7.41 & NSDAP, Offiziersgruppe \\
\hline \multirow[t]{3}{*}{ Oberlt. } & Charisius & Nov. 41 & NSADP, Offiziersgruppe \\
\hline & Reyher & Nov. 41 & Offiziersgruppe \\
\hline & Rücker & 5.8 .42 & NSDAP, Offiziersgruppe \\
\hline \multirow[t]{3}{*}{ Leutnant } & Einsiedel & 30.8 .43 & Stalingrad \\
\hline & Kehler & & Offiziersgruppe \\
\hline & Kügelgen & 19.8 .43 & Stalingrad \\
\hline Feldwebel & Stresow & & \\
\hline Uffz & Klein & 27.7 .41 & \\
\hline \multirow{6}{*}{$\begin{array}{l}\text { Obergefr. } \\
\text { Gefreiter }\end{array}$} & Luddeneit & 3.8 .41 & KJVD, übergelaufen, Antifa-Schule \\
\hline & Eschborn & & \\
\hline & Helmschrott & 16. 9.42 & \\
\hline & Kertzscher & Juli 1941 & Antifa-Schule \\
\hline & Krummel & & Antifa-Schule \\
\hline & Zippel & 4. 7.41 & KPD, übergelaufen, Antifa-Schule \\
\hline \multirow[t]{5}{*}{ Soldat } & $\operatorname{Sin} z$ & 24.6 .42 & KPD, übergelaufen, Antifa-Schule \\
\hline & Emendörfer & 17.1 .42 & KPD, übergelaufen, Antifa-Schule \\
\hline & Fleschhut & 31.7 .41 & Antifa-Schule \\
\hline & Keßler & 15.7 .41 & KJVD, Antifa-Schule \\
\hline & Kühn & & Antifa-Schule \\
\hline
\end{tabular}

Von kommunistischer Seite unterschrieben das Manifest des Nationalkomitees Anton Ackermann, Martha Arendsee, Johannes R. Becher, Willi Bredel, Wilhelm Florin, Edwin Hoernle, Hans Mahle, Wilhelm Pieck, Gustav Sobottka, Walter Ulbricht, Erich Weinert und Friedrich Wolf. Die Kriegsgefangenen Günther Kertzscher, Emil Krummel, Gerhard Krausnick, Herbert Stresow und der Kommunist Gustav von Wangenheim unterschrieben am 12. Juli das Gründungsmanifest nicht, wurden später aber - aus nicht nachvollziehbaren Gründen - dennoch zu seinen Mitgliedern gezählt ${ }^{59}$.

57 Wolff, Die erste Konferenz, S. 277 ff.; Robel, Antifa, S. $53 \mathrm{ff}$.

58 Charakteristiken des UPVI, GARF 9401/2/97, Bl. 218-224, ebenda Akte 269, Bl. 235-247.

59 Vgl. FD Nr. 1/1943 vom 19. 7. 1943. In seiner späteren Darstellung zählt Weinert (National- 
Um das Nationalkomitee wie vorgesehen im Juli 1943 gründen zu können, mußte man auf die kleine Gruppe der ersten Antifa-Aktivisten zurückgreifen ${ }^{60}$. Gemessen an der ursprünglichen Intention der sowjetischen Führung kam somit der eigentliche propagandistische Erfolg erst mit der Gründung des Bundes Deutscher Offiziere (BDO) im September 1943, mit dem es schließlich gelang, eine Reihe von höheren Offizieren und Generälen zur Mitarbeit zu gewinnen. Die Anleitungsstrukturen aber, in die das NKFD und später auch der BDO eingebunden wurden, entstanden im August mit der Schaffung des Instituts 99.

\section{Institut 99}

Die Vorbereitungen zur Gründung des Nationalkomitees wurden begleitet vom Aufbau eines „Büros des Komitees zur Erledigung der laufenden Arbeiten“, aus dem sich unter der Bezeichnung „Institut 99“ die Koordinierungsstelle für die gesamte politische Arbeit unter den Kriegsgefangenen entwickelte. Denn als seine Aufgaben wurden nicht nur die Anleitung des NKFD festgesetzt, sondern auch Zuständigkeiten, die weit darüber hinausgingen: „Zu den besonderen Aufgaben des Büros gehört: Die [Glav/PURKKA bei der politischen Aufklärungsarbeit unter den deutschen Offizieren und Soldaten an der Front und unter den deutschen Kriegsgefangenen zu unterstützen, insbesondere bei der Herausgabe der Zeitung, Flugblätter, Broschüren und bei der Schallplatten- und Lautsprecherpropaganda. Außerdem soll das Büro sich im Namen des Komitees mit eigenen Aufrufen an das deutsche Volk und an die deutschen Soldaten und Offiziere wenden, wozu auch das Radio systematisch ausgenutzt werden soll. (...) Das Büro führt die laufende Registratur des deutschen antifaschistischen Aktivs in den Kriegsgefangenenlagern und Kriegsgefangenenschulen im Einvernehmen und mit Hilfe der Verwaltung für die Kriegsgefangenenlager der NKWD [UPVI].“61 Der Aufbau des „Büros“ erfolgte Anfang August 1943 durch die Unterabteilung Information der 7. Verwaltung (Arthur Pieck) ${ }^{62}$. Dann übernahm „ein gutmütig dreinblickender Mann, der

komitee, S. 23) die fünf dennoch zu seinen Mitgliedern. Ebenso listet eine von Pieck angefertigte Aufstellung über die „Zusammensetzung des Nationalkomitees Freies Deutschland“ (SAPMO-BArch NY 4036/575, Bl. 18-20) die genannten fünf auf. Da auch eine Ende 1945 aufgestellte Liste der Mitglieder (SAPMO-BArch NY 4065/19, Bl. 6-8) die fünf nennt, kann angenommen werden, daß sie bei der Gründungsveranstaltung nicht unterzeichnen konnten - oder sollten -, aber dennoch als NKFD-Mitglieder anzusehen sind.

60 Vgl. Robel, Antifa, S. 75 f.; Einsiedel, Tagebuch der Versuchung, S. 62 und 66f.; siehe auch „Motive, die zur Gründung des BDO führten“, BArch Abt. Potsdam 90 KO 10/28, Bl. 30-31 RS.

61 „Vorschlag zur Bildung des deutschen Komitees zum Kampf gegen Hitlerkrieg und Nazityrannei" vom 27. 5. 1943, SAPMO-BArch NY 4036/575, Bl. 5.

62 Arthur Pieck war der Sohn Wilhelm Piecks. Seit 1938 besaß er die sowjetische Staatsangehörigkeit und trat am 8.7.1941 im Range eines Hauptmannes in die Rote Armee ein. Er war in der 7. Verwaltung vor allem im Zusammenhang mit dem Einsatz von Kriegsgefangenen als Frontpropaganisten tätig. Zusammen mit dem Frontstab der 1. Belorussischen Front marschierte er nach Berlin ein, wo er, nach seiner Demobilisierung, am 19.5. 1945 in den Magistrat gewählt wurde; Seleznev, Genosse politruk Artur Pik; Voßke, Ein Wegbereiter der deutsch-sowjetischen Freundschaft; Fischer, Arthur Pieck. 
sich ,Kozlov" nannte" die Leitung des Instituts ${ }^{63}$. Michail Vassilevič Kozlov war bis Ende 1945 Chef des Instituts 99. Danach übernahm die Leitung bis zur Auflösung des Instituts 99 zum 31. Dezember 1946 ein Funktionär namens Romanov. Über die Laufbahnen beider Institutsleiter ist nichts bekannt. Kozlov kam möglicherweise aus der Kaderabteilung der Komintern. In einem nicht realisierten Stellenplan für den projektierten Sonderapparat innerhalb der Geschäftsabteilung des ZK der VKP (b) war er als Abteilungsleiter vorgesehen 64 .

Der zweite Mann im Institut 99 war der Kaderleiter Stefan Nikiforovič Vorob'ev, bei dem es sich möglicherweise um den Major handelt, der beim UPVI die Registrierung antifaschistischer Kriegsgefangener geleitet hatte ${ }^{65}$. Die weiteren Mitarbeiter waren diejenigen Kominternfunktionäre, die auch schon vorher die politische Arbeit unter den Kriegsgefangenen organisiert hatten. Insofern war das Institut 99 personell die Fortsetzung der bisherigen Arbeit, aber mit dem organisatorisch entscheidenden Unterschied, daß die drei konkurrierenden Apparate von Komintern, UPVI und GlavPURKKA nun in einer gemeinsamen Institution zusammenarbeiteten. Das Institut 99 war damit nicht eine Fortsetzung der EKKI-Kommission, die an dem Widerstand von UPVI und GlavPURKKA gescheitert war, sondern stellte den Versuch dar, endlich eine wirksame Koordinierung der politischen Arbeit unter den Kriegsgefangenen herzustellen ${ }^{66}$.

Die Struktur des Instituts 99 glich der der Institute 100 und 205. Es verfügte über eine eigenständige Institutsleitung mit Sekretariat und Kaderverwaltung. Das Institut 99 stellte einen eigenen Etat auf und konnte somit den Mitarbeitern Einkommen und durch den ebenfalls institutseigenen Kantinenbetrieb Verpflegung bieten ${ }^{67}$. Ansonsten gab es drei Abteilungen: der zivile Teil des NKFD mit seinen beiden Redaktionen (Zeitung und Radiosender), die Redaktionen der Zeitungen für die italienischen, ungarischen und rumänischen Kriegsgefangenen und die Abteilung für die Antifa-Schulen. Zum 1. Oktober 1943 waren insgesamt 94 Personen im Institut 99 angestellt. Ein Jahr später, zum 1. September 1944, war der Mitarbeiterstab auf 140 Personen angewachsen ${ }^{68}$. Der konspirative Tarnname entsprach der verdeckten Arbeitsweise. So waren die Mitarbeiter gehalten, im Umgang mit sowjetischen Dienststellen nur die - inhaltlich nichtssagende - Bezeichnung „Institut $99^{“}$ zu verwenden

63 Leonhard, Revolution, S. $283 \mathrm{f}$.

${ }^{64}$ RGASPI 495/73/208, Bl. 118. Huber (The Cadre Department, S. 146) nennt einen Kozlov als Abteilungsleiter der Kaderabteilung. $\mathrm{Da}$ es sich jedoch um einen häufigen russischen $\mathrm{Fa}_{\mathrm{a}}$ miliennamen handelt, sind Verwechslungen nicht ausgeschlossen.

65 Leonhard, Revolution, S. 284. Vorob'ev ist ebenfalls ein sehr gebräuchlicher russischer Familienname, so daß bloße Namensgleichheit wenig aussagt. Allerdings wird in den Unterlagen des Politstellvertreters des UPVI, Oberst Jakovec, ein Major Vorob'ev als sein Stellvertreter genannt, und es wäre angesichts der Streitigkeiten zwischen UPVI und EKKI-Kommission um die Kaderregistratur plausibel, wenn man auf die hier vermutete Personallösung zurückgegriffen hätte.

66 Siehe demgegenüber Reschin, Feldmarschall im Kreuzverhör, S. 55 f.; ders., General zwischen den Fronten, S. $8 \mathrm{ff}$.

67 Gehalt und gesicherte Ernährung waren für die Funktionäre überlebenswichtig, denn der Verbleib in Moskau war an einen festen Arbeitsplatz gebunden. Die Ernährung über Kantinenspeisung einer ZK-Einrichtung dürfte weit über dem Ernährungsniveau der sowjetischen Bevölkerung gelegen haben.

68 Stellenplan des Instituts 99 zum 1. 10. 1943 und 1. 9. 1944, RGASPI 578/5/4, Bl. 5-7 und ebenda 495/77/39, Bl. 1-6; siehe Anlage Nr. 1. 
und keine Angaben zu ihrer Tätigkeit zu machen. Auch den kriegsgefangenen NKFD-Mitgliedern blieb die Existenz des Instituts 99 weitestgehend verborgen. Im Lager Lunevo wußte man zwar vom „Stadtkomitee“, in dem die kommunistischen Komiteemitglieder arbeiteten, aber daß dahinter ein vollständiger, in die sowjetische Bürokratie integrierter Apparat steckte, dürfte den wenigsten klar gewesen sein. Leonhard, damals Mitarbeiter des Instituts 99, urteilte: „Die meisten Mitglieder des Nationalkomitees in Lunevo wußten wahrscheinlich von der Existenz des ,Stadtkomitees', waren aber über seine Tätigkeit nicht informiert. Es dauerte nicht lange, bis ich erkannte, daß die eigentliche politische Redaktionsarbeit mehr in diesen Räumen als am offiziellen Sitz des Nationalkomitees [in Lunevo] geleistet wurde." 69

Ein "Stadtkomitee" war in der Organisationsstruktur des Nationalkomitees nicht vorgesehen. Nach den Statuten des NKFD bildete das Plenum das oberste Entscheidungsgremium, zu dem alle Komiteemitglieder mindestens einmal im Monat in sogenannten Vollsitzungen zusammenkommen sollten. Zwischen den Vollsitzungen fungierte der Geschäftsführende Ausschuß als Exekutive. In ihm waren anfangs paritätisch zwei Kommunisten (Weinert, Ulbricht) und zwei Kriegsgefangene (Zippel, Hadermann) vertreten. Nach dem Zusammenschluß des NKFD mit dem BDO wurde er um sechs Kriegsgefangene erweitert und bestand nun aus den Emigranten Weinert und Ulbricht sowie den kriegsgefangenen NKFD-Mitgliedern Hadermann, Reyher, Rücker, Zippel und den BDO-Mitgliedern Korfes, Lattmann, Steidle und van Hooven. Zusätzlich wurde eine operative Abteilung des Geschäftsführenden Ausschusses (Ulbricht, Reyher, Steidle, van Hooven, Zippel) eingesetzt. Das Präsidium des Nationalkomitees - Weinert als Präsident und die Kriegsgefangenen von Seydlitz, von Daniels, Emendörfer, Hetz und Einsiedel als Vizepräsidenten - arbeitete jedoch nicht als Körperschaft gleichberechtigter Mitglieder, sondern wurde ausschließlich vom Präsidenten Weinert geleitet ${ }^{70}$. In der laufenden Arbeit des Nationalkomitees lagen die meisten Kompetenzen beim Präsidenten Weinert bzw. bei der operativen Abteilung unter Ulbricht. Beide arbeiteten im Institut 99. So wurden die im Namen des Nationalkomitees in die Kriegsgefangenenlager und an die Front als Propagandisten entsandten „Lager- und Frontbevollmächtigten des NKFD“ ausschließlich vom Institut 99 eingesetzt, dem sie auch Rechenschaft schuldig waren. Der operativen Abteilung, d.h. dem Institut 99, unterstanden zudem die „Fachgruppen des NKFD“71. Es ist daher nicht erstaunlich, $\mathrm{daß}$ die Willensbildung innerhalb des Nationalkomitees als „weitgehend von Kommunisten gesteuert" bezeichnet wurde: „Zumindest wird deutlich, daß die Gremien, an denen die anfangs argwöhnischen Militärs beteiligt waren (Vollsitzung, Geschäftsführender Ausschuß), bei Eigenmächtigkeit der räumlich von ihr getrennt arbeitenden operativen Leitung allenfalls korrigierende Maßnahmen durchsetzen konnte, daß nichtkommunistische Initiativen jedoch bei ihrer Realisierung auf die Zustimmung der Kommunisten angewiesen waren." 72

${ }^{69}$ Leonhard, Revolution, S. 285. In der Erinnerungsliteratur der kriegsgefangenen NKFDMitglieder geht lediglich Einsiedel (Tagebuch der Versuchung, S. 76) auf das Büro ein, „von dem aus die Organisationsfragen mit den Sowjets gelöst werden".

70 Notizen Piecks „Daten der Bewegung“, SAPMO-BArch NY 4036/575, Bl. 248.

71 Weinert, Nationalkomitee, S. 30 f.; vgl. Organisationsschema des NKFD, in: Vsevolodov, Vzaimodejstvie politorganov, Anlage Nr. 4.

72 Sywottek, Deutsche Volksdemokratie, S. 125. 
Der Willensbildungsprozeß im Nationalkomitee erfolgte über kontinuierlich durchgeführte Absprachen im Institut 99, die Wilhelm Pieck in seinen Notizen „Zivilsektorsitzungen“ nannte. Diese Sitzungen dienten zur Abstimmung der allgemeinen politischen Linie, insbesondere der Propaganda, und zur Klärung von Einzelfragen, wobei den Personalentscheidungen („Kaderfragen“) besonderes Gewicht zukam. Der Personenkreis, der zu diesen Sitzungen zusammenkam, läßt sich für die Zeit bis März 1944 nicht eindeutig rekonstruieren. Wahrscheinlich waren es die hauptamtlichen NKFD-Funktionäre (Weinert, Ulbricht und als Redaktionsleiter Ackermann und Herrnstadt), KPD-Chef Pieck, der Institutschef Kozlov und Vertreter der 7. Verwaltung (Burcev, Braginskij) sowie eventuell ein Mitarbeiter des UPVI. Von März 1944 bis Mai 1945 trafen sich auf den Zivilsektorsitzungen im Institut 99: Kozlov, Ulbricht, Ackermann, Herrnstadt bzw. als sein Vertreter Maron, Weinert, Pieck, Braginskij, Burcev und Gerö (Zensor der 7. Verwaltung für die Redaktionen des NKFD); darüber hinaus Švec (operative Abteilung des UPVI) und bei wichtigeren Anlässen Manuil'skij. Durch die „Zivilsektorsitzungen“ waren UPVI und GlavPURKKA unmittelbar an der Arbeit des Instituts 99 beteiligt, das zudem über Manuil'skij in die Politik des Rates für militärpolitische Propaganda eingebunden wurde. In der laufenden Arbeit wurden - vermutlich zur Bestätigung - die Beschlußprotokolle des Instituts 99 dem OMI vorgelegt ${ }^{73}$.

Die erste Sitzung des „Zivilsektors“ fand während der zweiten Vollsitzung des NKFD - des nominellen Entscheidungsgremiums - statt. Bevor das zweitägige Plenum am Folgetag fortgesetzt wurde, wurden am Morgen des 20. Juli die vom NKFD gefaßten Beschlüsse korrigiert: Major Homann war zum Koordinator für die NKFD-Bevollmächtigten in den Kriegsgefangenenlagern gewählt worden, der Zivilsektor aber hob diesen Beschluß auf, „weil unzweckmäßig"74. Über die Beweggründe dieser Korrektur kann nur gemutmaßt werden, aber es ist anzunehmen, daß die Koordinierung der NKFD-Bevollmächtigten ausschließlich in der Hand des Instituts 99 bleiben sollte. Allerdings rief das derart ungehemmte Eingreifen in die Beschlüsse der Vollsitzung den Protest der Betroffenen hervor ${ }^{75}$. Um in $\mathrm{Zu}-$ kunft Spannungen mit den Kriegsgefangenen zu vermeiden, wurden die Zivilsektorsitzungen im weiteren anberaumt, bevor das Plenum des NKFD zusammenkam. Fanden von Juli 1943 bis Februar 1944 sieben Vollsitzungen statt, so kam der Zivilsektor in dieser Zeit elf Mal zusammen ${ }^{76}$. Damit unterlagen die Entscheidungen des Plenums des NKFD immer einer direkten, anfangs noch plump ausgeübten Kontrolle durch das Institut 99. Ab März 1944 fanden die Sitzungen im wöchentlichen Turnus statt, um die Abstimmung zwischen Institut 99, 7. Verwaltung und operativer Abteilung des UPVI zu optimieren. Hinsichtlich des Meinungsbildungsprozes-

73 Siehe die Beschlußprotokolle des Instituts 99; besonders zu beachten auch die Fundorte: RGVA/K 88/3/1 (UPVI-Abteilung für die Antifa), RGASPI 495/77/46 (Büro Dimitrov) und ebenda 17/128, Akte 787 und 788 (OMI). Das Archiv des sowjetischen Verteidigungsministeriums (GlavPURKKA) ist leider für die Forschung nicht zugänglich.

74 Notizen Piecks „Daten der Bewegung“, SAPMO-BArch NY 4036/575, Bl. 245.

75 Ebenda, Bl. 245-246: „Erklärung von Hetz im Namen von Hetz, Stößlein, Homann (3 Majore) - Protest gegen verschiedene Vorgänge, mit denen nicht einverstanden - Demission Verstoß gegen die Demokratic - Sitzung unterbrochen - nach Wiedereröffnung Zurückziehung der Unterschrift unter Erklärung, aber Demission, später auch Demission aufgegeben."

76 Ebenda, Bl. 243-253. 
ses ist zudem von Bedeutung, daß die Leitung der KPD in der Besetzung Pieck, Ulbricht, Ackermann und Florin ${ }^{77}$ regelmäßig zusammenkam. Aufgrund der personellen Verquickungen werden diese "Leitungssitzungen“ der KPD - zu denen Pieck ebenfalls ausführlich Notizen anfertigte - auch Auswirkungen auf die Arbeit im Institut 99 gehabt haben. Wichtiger aber war, daß nach der Auflösung des Rates für militärpolitische Propaganda im Juli 1944 die KPD-Führung bei konkreten Anlässen in das ZK der VKP (b) zitiert wurde, wo sie in „Besprechungen bei Dimitrov" - so die Pieck-Notizen - direkte sowjetische Anweisungen erhielt.

Aus dem Geflecht unterschiedlicher Beratungszirkel ergibt sich quellenkritisch das Problem, daß in der bruchstückhaften Überlieferung der Quellen das Institut 99 nur selten explizit erwähnt wird. Vor allem durch die Notizen Piecks entsteht der Eindruck, die KPD habe direkt mit der sowjetischen Führung über deutschlandpolitische Entscheidungen im Zusammenhang mit dem Nationalkomitee „Freies Deutschland" verhandelt. Aber die KPD-Funktionäre waren nur wegen ihrer Mitarbeit im Institut 99 an der Durchführung der sowjetischen Entscheidungen beteiligt. Ihr Anteil an der Entscheidungsfindung selbst war sehr gering. Die konzeptionellen Fragen des NKFD wurden auf ZK-Ebene von Manuil'skij bzw. Dimitrov entschieden und bei der sowjetischen Führung die erforderlichen Beschlüsse erwirkt. Die Ausarbeitung der Beschlußvorlagen erfolgte zwar durch die KPD-Führung, die aber entweder durch die Sitzungen im Institut 99 oder aber direkt durch die Anweisungen Dimitrovs instruiert wurde. Die daraus resultierende Politik wurde in einem komplizierten Rückkopplungsmechanismus aus Vorlage, Anweisung, Überarbeitung und Entscheidung gestaltet. Denn auch wenn das NKFD unmittelbar durch das Institut 99 gesteuert wurde, so war das Institut selbst doch nur ausführendes Organ, das sich an übergeordneten deutschlandpolitischen Konzeptionen der sowjetischen Führung orientieren mußte.

77 Wilhelm Florin starb am 5. 7. 1944 in Moskau; „Nach Hitler kommen wir“, S. 405. 